\title{
Hardware structure of a surveillance system for strategic objectives
}

\author{
Victor Constantin ${ }^{1 *}$, Mihai Avram ${ }^{1}$, Constantin Bucsan ${ }^{1}$, and Alexandru Vlasceanu ${ }^{1}$ \\ ${ }^{1}$ University Politehnica of Bucharest, Splaiul Independentei 313, Bucharest, Romania
}

\begin{abstract}
The paper presents the hardware structure of a system for the surveillance of strategic objectives. The proposed system consists of several mobile robots patrolling along a perimeter, identifying potential intruders. The information obtained is transmitted to both the mobile robots and a fixed control system, which may decide to get more detailed information about the event, sending a quadcopter in the area with a high-resolution infrared camera, and a radar sensor. It communicates to the control system all the information obtained, and these will lead to a decision.
\end{abstract}

\section{Introduction}

Unmanned (UAV) vehicles can be used to monitor and monitor large areas of land, but have the disadvantage that sensors mounted on the UAV are usually limited to the exact location of the targets. To solve this problem, unmanned land-based vehicles (UGVs) are used[8]. They can be used to accurately locate ground targets but have the disadvantage that they cannot move quickly and can encounter a number of obstacles such as buildings, cars, or any other ground-level object [5]. In this article, some architectures of UAV and UGV based monitoring systems are presented.

\section{State of the art}

A surveillance structure based on UAV and UGV is presented in the paper [1]. The aircraft is a Piper Cub J3 model with a wing width of approximately $2.7 \mathrm{~m}$. The engine has a $3.5 \mathrm{cp}$ power, providing a maximum cruising speed of about $30 \mathrm{~m} / \mathrm{s}$ at altitudes of up to $1500 \mathrm{~m}$ and a flight autonomy of 15-20 min. The aircraft / engine combination allows for the loading of significant payloads on board. Figure 1 shows cassettes that have been installed beneath each side of the wing, containing high resolution cameras and inertial measurement units (IMUs), as well as sensors, beacons, laser for marking possible threats, etc. Among the features of this system are a built-in onboard computer, in-built measurement system, MicroStrain 3DM-G type, Global Positioning System (GPS), DragonFly camera, IMU Pod Custom Camera, including inertial measurement system and camera surveillance, mounted on the same support.

\footnotetext{
${ }^{*}$ Corresponding author: victor.constantin@upb.ro
} 


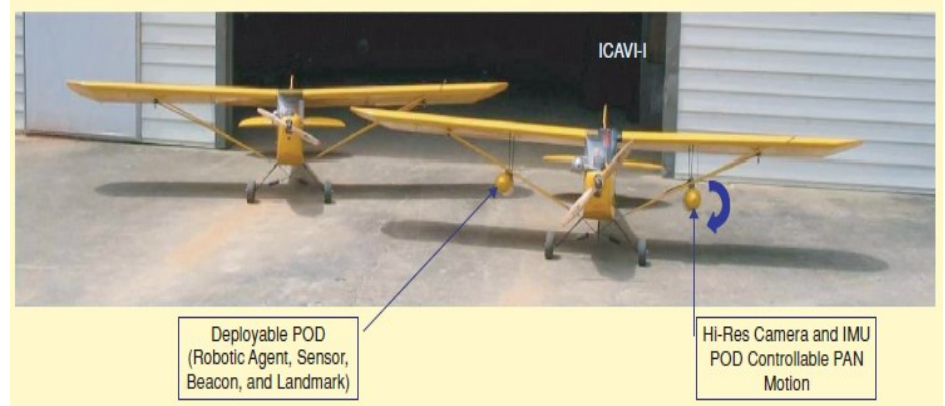

Fig 1. Piper J3 Cub equipped with mobile robot deployment support [1]

Each UAV is controlled by a customized avionics system. The avionics system comprises a base autopilot, a set of sensors, which also includes GPS and an IMU consisting of three gyroscopes, three accelerometers and two pressure ports, one for barometric altitude and one for speed.

The ground vehicles, shown in Figure 2, are fully modified traction commercial vehicles equipped with onboard computers, firewire stereo cameras, GPS and odometer and inertial sensors. Communication between terrestrial vehicles and the base station of the aircraft platform is via an ad-hoc 802.11 b network.

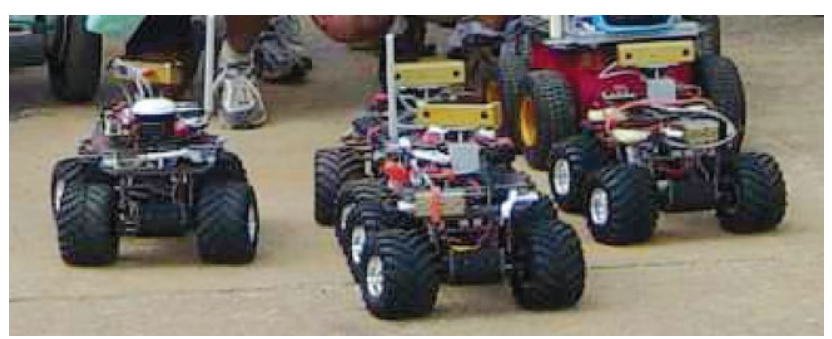

Fig 2. UGV equipped with smart devices[1]

In the paper [2] it is proposed to develop an environmental monitoring system on the surface extended and long-term, using multi-standalone robots (UGVs). The concept of the planned monitoring system is to use low cost mobile robots (Figure 3). Each robot has limited capability, but the system as a whole is able to cope with unexpected situations, sharing the experience of each robot with wireless communications. When a robot is deactivated as a result of an unexpected situation, the system will temporarily abandon the robot and the other robots will compensate for the loss of the robot.

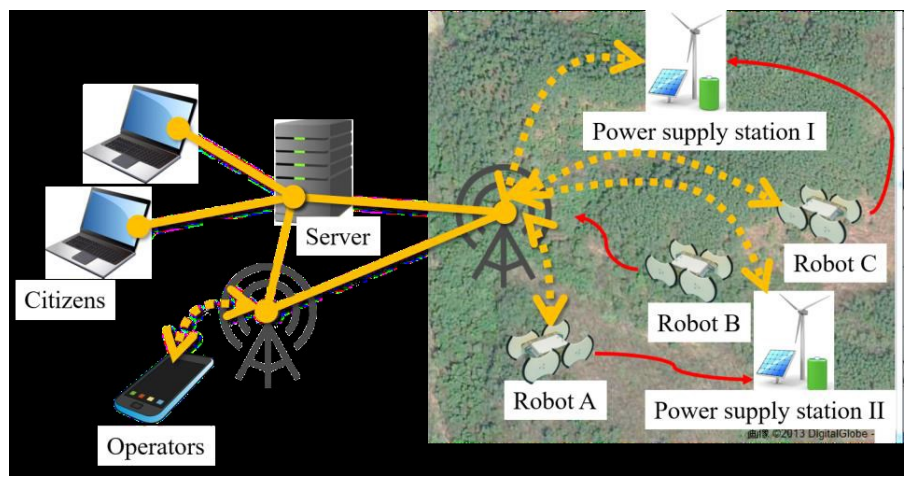

Fig 3. Mobile robot system for objective tracking [2] 
In the above figure, mobile robots move autonomously and collect environmental data through co-operation between them. The robot moves around its power station as a base point and returns to the station when the battery level becomes low. The yellow line represents the communications system and the red line shows the robotic route.

Robot design has focused on delivering high locomotion performance at a low cost. The robot must move on rough terrain such as forest areas and be sufficiently durable to deal with unexpected events during long-term operation.

Due to the multitude of UV applications, various researchers have used in the past years agent-based simulation (ABS), a modeling technique used to simulate a real system (vehicles, people, interactions) in an integrated environment. These agent-based models have been used in various experiments to investigate simulated real-system behavior in different settings to gain benefits such as cost savings and performance improvements (time, distance, energy consumption).

Incorporating real hardware using hardware-in-the-loop (HILS) simulation techniques is an important step in achieving system-level development and real-time testing, taking into account various aspects such as time, cost and safety. Various studies have been carried out to apply HILS-based agents for UAV and UGV systems.

In [3] the authors propose a HILS-based agent for crowd surveillance and control. The ABS is integrated with a real UAV through the hardware interface to perform both monitoring functions (updating sensor data) and command execution for the UAV / UGV system control.

Using an agent-based hardware-in-the-loop simulation (HILS), one can study the characteristics of the various control architectures (centralized or distributed) for unmanned aerial vehicle (UAV) and unmanned land-based (UGV) performing collaborative supervision and crowd control [4], Figure 4.

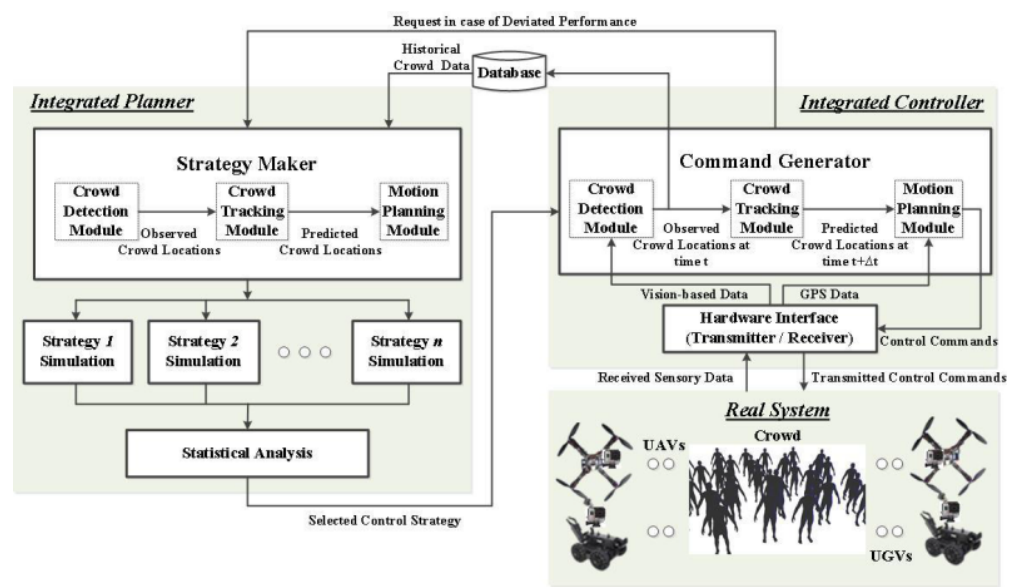

Fig 4. Simulation-based architecture for crowd control [3]

The UAV platform chosen to perform such an experiment is a quadcopter (Figure 5a). The system is optimized for the smallest cost and flexibility to carry various kinds of useful tasks, including a surveillance camera and a powerful built-in computer for data processing. The flight microcontroller selected for the UAV platform is ArduPilotMega (APM) 2.5, an autopilot platform. This autopilot is based on the Arduino Mega 2560 and features an integrated inertial measurement unit (IMU) that measures acceleration, rotation speed and magnetic field direction.

The projected UGV platform (Figure 5b) is based on a four-wheel-drive platform. It is equipped with a microcontroller to process IMU data, serial commands and user input on a 
2.4 GHz radio frequency and sends control signals to change the direction and speed of the UGV. One of the limitations for the first UGV platform is the lack of fine control of motion that is required for operating the system in the real world.

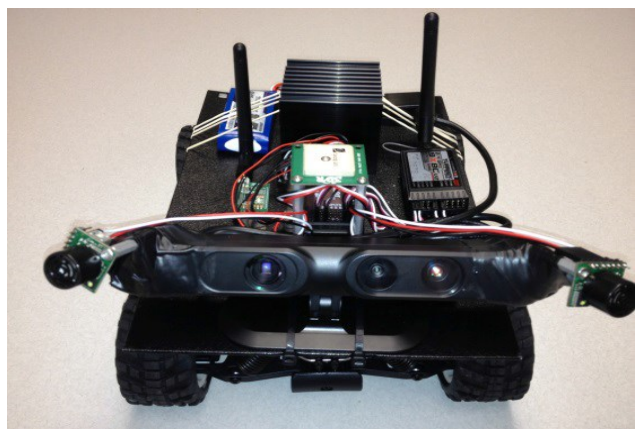

Fig. 5a. UGV platform

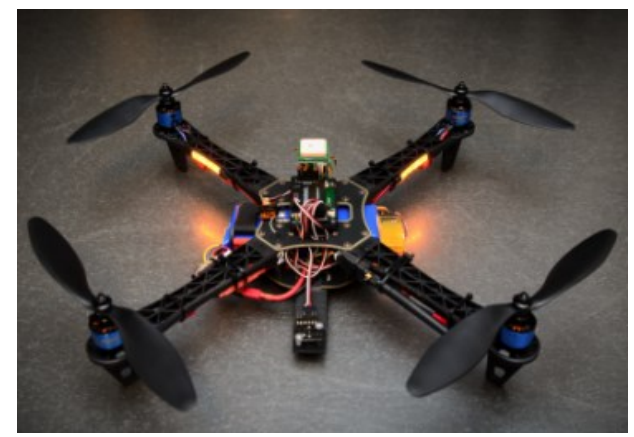

Fig. 5b. UAV platform

In [6] the authors propose designing differential architectures to monitor intelligent cities and oceans by several UAVs.

Thus, for smart city monitoring, it is proposed to create a rigid framework based on plans, which takes into account several practical scenarios according to specific objectives, namely:

- Rapid detection and reporting of disasters;

- Tracking a possible fugitive;

- Delivery of emergency items and disaster recovery.

- Prevention and detection of terrorist threats;

- Sustainable surveillance and patrolling of high accident rates.

Based on these scenarios, a dense model is proposed on a horizontal basis that, to support the UAV network, allows both the installation of UGSs and the use of the public transport system. Handling UAVs, the proposed architecture for intelligent city is based on a combination of autonomous flight and controlled maneuvers by authorized users. UAVs can operate independent flights to achieve specific tasks, including travel to certain locations and takeoff / landing UGSs and public buses.

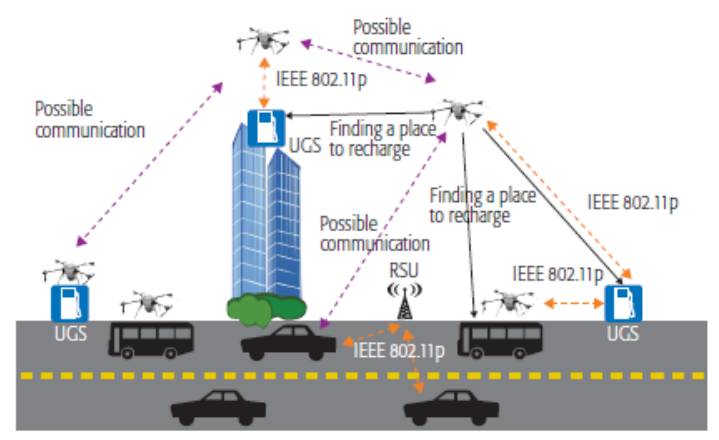

Fig. 6. Smart city monitoring architecture [6]

When events are announced by the control center, the system makes a list of UAVs that may be based on current information including UAV locations and their current battery status as well as map information covering the type area, obstacles, etc. Thus, the system decides which UAVs should go to the location of the specific event, on an efficient trajectory, using the current map information to minimize the delay. 


\section{Proposed system}

The proposed system consists of a number of $\mathrm{N}$ autonomous mobile robots capable of crossing rugged terrain, connected via a $4 \mathrm{G}$, long-range Wi-Fi communication protocol. They are tasked with monitoring an area according to a predetermined trajectory, the objective being monitored by means of temperature, humidity sensors, presence sensors, sound, etc. The positioning of the robots is based on a GPS system and the assignment of tasks is done according to a predetermined algorithm. Robots communicate based on a decentralized architecture, and in the event of an unknown event, this change is communicated to all mobile $\mathrm{N}$ robots. If an algorithm determines that it is necessary to investigate the perturbation at the reported coordinates, a quadcopter robot equipped with long sensors more performing.

This solution has the advantage of reduced cost, high energy autonomy and redundancy. The schematic diagram of the proposed system is shown in figure 7.

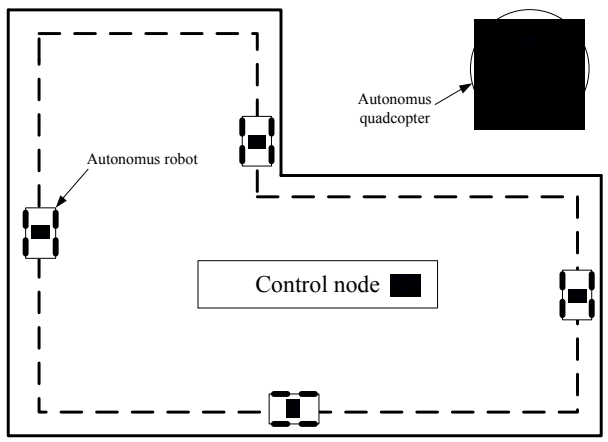

Fig. 7. Basic schematic of the prosed surveillance system

In the proposed version, all robots have the same configuration. The platform is driven by a geared DC motor for each of the four wheels. The motor control is implemented using a microcontroller and a microcomputer configuration. The robot movement control algorithm is implemented at the microcontroller level and the rest of the operations (communication, task division, data storage and distribution) are done at the microcomputer level.

Robot control is performed with an application developed by the authors in the Python programming language. It uses the TCP protocol for robot communication and Websocket protocol for reporting data to a user.

Interacting with the system (viewing data, controlling one or more robots) is done through a web page stored at the level of each robot and implemented using a Flask microframework that allows direct interaction with the Python. The user interface allows for simple operations such as direction control, speed control, selecting a particular robot, shutting down and starting the system, etc.

The system will also feature a method for imposing certain behaviours from both the autonomous robots and quadcopters, such as certain trajectories, stopping points, which data to collect based on the sensors that they are equipped with, etc.

Work is based on a previous model of the system, one that does not include the quadcopter [9], figure 8. 


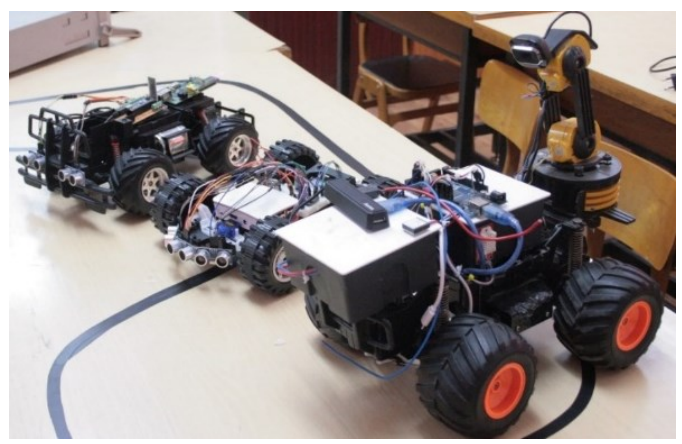

Fig. 8. Platforms designed for system testing

Current design includes identical platforms for all of the robots so that tasks can be more easily distributed between them. A quadcopter model is also being tested (figure 9).

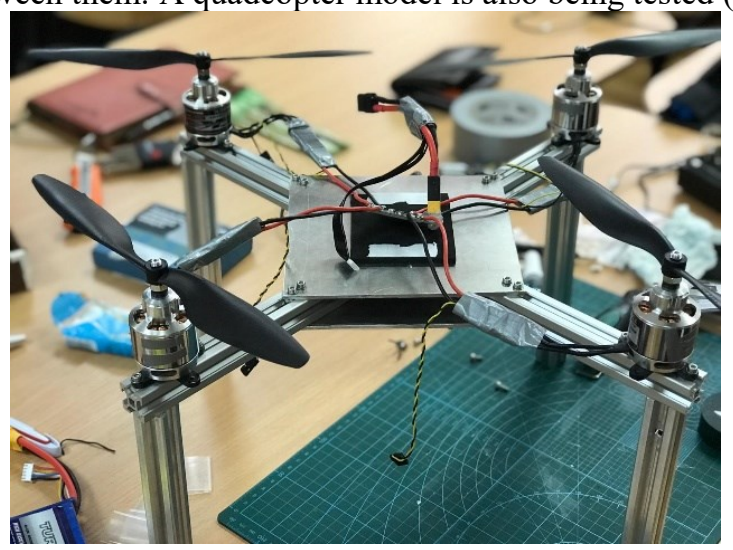

Fig. 9. Quadcopter testing model

On the mechanical side, a method for 3D printing the robots is being proposed, based on the mechanical and electrical properties of the materials used in paper [7].

\section{Conclusions}

The paper shows a study of the state of the art of current robot based surveillance systems, with emphasis on those composed from both terrestrial and aerial autonomous robots. A method and possible architecture is proposed by the authors based on the study and an opensource module will be further developed for implementing the system. Further testing of the proposed platform will be performed in future work, both along the lines of software and hardware development. Also, a carrier system for the robots will be taken into consideration, by means of the proposed quadcopter systems.

\section{References}

1. B. Grocholsky, J. Keller, V. Kumar, G. Pappas, IEEE RAM, 3 (2006)

2. K. Tanaka, O. Yuya, I. Hiroyuki, K. Daisu, IEEE AIM - Munich, Germania (2017)

3. A. M. Khaleghi, D. Xu, A. Lobos, S. Minaeian, - IEEE WSC (2013)

4. A. M. Khaleghi, D. Xu, S. Minaeian, M. Li, Y. Yuan,

5. J. Liu, Y. J Son - Proceedings ISER Conference (2014)

6. K. Tanaka, H. Ishii, S. Kinoshita, Q. Shi, - IEEE ROBIO, 1763-1768 (2014) 
7. H. Kim, L. Mokdad, B. O. Jalel, IEEE CM, 56, 98-104 (2013)

8. A. Spanu, D. Besnea, I. Voiculescu, MP, 55, Issue 2, 141-145 (2018)

9. V. Constantin, C.I. Rizescu, M. Ciocan, D. Rizescu, ASIR (2018) 